\title{
Computational procedures for stochastic multi-echelon production systems
}

\author{
G.J. van Houtum \\ Eindhoven University of Technology, Department of Mathemattcs and Computing Science, \\ PO Box 513, 5600 MD Eindhoven. The Netherlands \\ and W.H.M. Zijm \\ University of Twente, Department of Mechanical Engineering. P O. Box 217,7500 AE Enschede, The Netherlands
}

\begin{abstract}
This paper is concerned with the numerical evaluation of multi-echelon production systems. Each stage requires a fixed predetermined leadtime; furthermore, we assume a stochastic, stationary et.d-tıme demand process. In a previous paper, we have developed an analytical framework for determining optımal control policies for such systems under an average cost criterion

The current paper is based on this analytical theory but discusses computatıonal aspects, in particular for serial and assembly systems $A$ hierarchical (exact) decomposition of these systems can be obtained by considering echelon stocks and by transforming penalty and holding costs accordingly. The one-dimensional problemis arising after this decomposition however involve incomplete convolutions of distribution functions, which are only recursively defined. We develop numerical procedures for analyzing these incomplete convolutions; these procedures are based on approximations of distribution functions by mixtures of Erlang distributions Combıning the analytically obtained (exact) decomposition results with these numerical procedures enables us to quickly determine optımal order-up-to levels for all stages. Moreover, expressions for the customer service level of such a mult1-stage system are obtained, yielding the possibility to determine policies which minimize average inventory holding costs, given a service level constraint
\end{abstract}

\section{Introduction}

In this paper we are concerned with the planning and control of the materials flow in a production chain. At the beginning of the chain raw materials and compenents are ordered from outside suppliers. Upon arrival these materials and components may be used immediately in a subsequent manufacturing stage if needed, or else may be stored in a component store. In particular when several components have to be assembled in one subassembly or final product, it may happen that components have to be temporarily stored since one other critical component is missing. After proceeding through a number of manufacturing stages, final products are stored in a factory warehouse from thich they are sold to the market.

In controllng such a chair of purchasing and manufacturing/assembly activities, one geiterally tries to balance a desired customer service level against reasonable production and inventory holding costs. This has been our ultimate goal as well; however, we start wirh analyzing the system under a $\mathrm{F}$.re cost criterion and later show how to translate the results to a model with both costs and service degrees. A central role in the analysis will be played by the concept of echelon stock, originally introduced by Clark [1] and exploited in a famous paper by Clark and Scarf [2] to establish the optimality of echelon stock based policies in a setial inventory system (in a discounted cost framework). Under certain assumptions, they have established an exact decomposition of the muiti-echelon serial system into a series of single stock systems (each associated with the echelon stock of a certain stage). Schmidt and Nahmias [3] extended their approach io a twa stage assembly system in which only two components are assem- 
bled in one final product, again under discounted cost assumptions. The analysis in these papers unfortunately leads to somewhat cumbersome formulas while the work of Schmidt and Nahmias seems not to be generalizable to systems with more than two components. In an average cost framework, Eppen and Schrage [4] have presented a thorough analysis of the stockless depot distribution system (one depot, which only acts as a pure distribution centre, and a number of end-time warehousus). A large number of authors have further contributed to the analysis of distribution systems (e.g. $[5,6]$ ).

Langenhoff and Zijm [7] have unified and extendid the above contributions in one general framework, which establishes exact decomposition results for arbitrary logistics chains in which assembly systems (with an arbitrary number of components), serial and distribution systems may all be present. They study multi-echelon production/distribution systems under an average cost criterion. The linear penalty and holding costs are transformed into appropriate echelon cost functions which form the basis of the (exact) decomposition results. As Clark and Scarf [2], they come up with artificial penalty cost functions at each echelon, which reflect the unability of a certain installation to satisfy downstream demand; due to the average cost structure these functions however are much less complicated than in the discounted cost analysis. Moreover, it can be shown that assembly systems can be transformed into almost purely serial systems, allowing for an almost direct application of the results for these serial systems to assembly systems as well (a similar result was recently established by Rosilng [8]). For distribution systems, the results parallel those of Eppen and Schrage [4], although Langenhoff and Zijm [7] also consider the case in which the central depot may hold stock.

The current paper is based on the analytical theory presented in Langenhoff and $\mathrm{Zijm} \mathrm{["i]} \mathrm{but} \mathrm{dis-}$ cusses numerical aspects. Although the eract decomposition results reviewed above reduces the analysis of a complex multi-dimensional problem to the analysis of a series of one-dimensional problems, the latter are not necessarily easy to handle. The one-dimensional functions which have to be minimized often involve incomplete convolutions of demand distribution functions; moreover, these functions are only recursively defined. We approximate these functions by matching mixtures of Erlang distributions to their first two moments (see e.g. [9, pp. 397-400]). These matchings have betn shown to perform extremely well, even when approximating convolutions of distributions in which one of the distributions has a positive probability mass at one point [10]. In between we note that matching distributions to the first two moments makes sense anyhow; often one does not have any further knowledge on the shape of the original distributions, in particular when forecasting market demand.

Bused on these al "oroximations, we develop numerical procedures for minimizing the singlt echelon cost functions arising after decomposition of the original muiti-echelon system. For ease of presenta. tion, and to avoid additional complexities, we restrict ourselves to production systems with serial and assembly structures. Briefly, we indicate also the possibility to anciyze multi-stage systems under a service level constraim. For production systems, the combination of the analytical theory discussed in [7] with the numerical procedures developed here enables us to analyze fairly complex systems in only a few seconds on an IBM-compatible PC/AT.

We conclude this section with an outline of the contents of the paper. In Section 2, we review the results of Langenhoff and Zijm [7] on the decomposition of general multi-echelon production/distribution systems, with an emphasis on serial structures. In Section 3, we discuss preliminaries on the approximation of incomplete convolutions by mixtures of Erlang distributions. Section 4 shows how this approximation technique can be used to numerically analyze the most simple multi-echelon system:: the two-stage serial system. In Section 5 we present results and algorithms for general serial systenis. Section 6 briefly discusses the extension to assernbly systems. Section 7 previews future work on distribution and finite capacity production systems.

\section{Fundameatal decomposition results for mult.-echelon systems}

In this scetion we seview the exact decomposition results derived by Langenhoff and Zijm [7]. Thro"ghout this pajn., bis assume that demand originates at the lowest installations only (i.e. the in- 
stallations at the downstream side of the logistic chain). We assume that all excess demand is backlogged which permits us to neglect any variable production or distribution costs. In addition, we do not consider fixed production and distribution costs. For a discussion on these assumptions as well as remarks on relaxation of some of them, see [7].

The echelon stock of a given installation includes all stock at that installation plus in transit to or on hand at any inet tit tion down'tream minus the backlogs at the most downstream installations (which do not have a suc iessor). Tiue chain under consideration is called the echelon. An echelon stock may Le negativs, indicating that the backlogs are larger than the total inventory in that echelon. Note that the echelon stock, associated with a component store, includes components on hand in that store, plus all components on hand at and in transit to any downstream installation, no matter whether these components have been used already in assembled products, minus possible backlogs. The echelon inventory position finally denotes the echelon stock plus the materials already ordered but not yet available at the highest (most upstream) installations. When an echelon consists of one installation, this definition coincides with the one given in [11]. Echelons are numbered according to the highest installation in thast echelon.

Consider first a serial system which consists of $N$ installations. Installation 1 is the most downstream installation tro $r$ which products are sold to the market. Products present at installation $n$ or in transfer between eche'on $n$ and echelon $n-1$ are charged at a rate $\sum_{k=n}^{N}\left(h_{k}\right)$ per unit of product per period, $A$ penalty cost $p$ per unit of product and per period is incurred if the lowest echelon $l$ is unable to meet market demand. Both penalty and inventory costs are charged at the end of a period.

Let $F_{l}$ denote the distribution of the $l$-period cumulative demand, for all $l$. If $l=1$, we suppress the index. Furthermore, $l_{n}$ denotes the delivery leadtime for goods ordered by installation $\boldsymbol{n}$ from the preceding installation if these goods are available $(n=1,2, \ldots, N-1)$ or from the (infinite capacity) initial supplier $(n=N)$. Now, transform the above defined costs into echelon holding and penalty cost functions, as follows:

$$
\begin{aligned}
& L_{1}\left(x_{1}\right)=h_{1} \int_{0}^{\infty}\left(x_{1}-u\right) \mathrm{d} F(u)+\left(p+\sum_{n=1}^{N}\left(h_{n}\right)\right) \int_{n 1}^{\infty}\left(u-x_{1}\right) \mathrm{d} F(u) \text { for all } x_{1}, \\
& L_{n}\left(x_{n}\right)=h_{n} \int_{0}^{\infty}\left(x_{n}-u\right) \mathrm{d} F(u) \text { for all } x_{n}(n=2, \ldots, N) .
\end{aligned}
$$

Define $\mu$ as the average demand per period. The the functions $L_{r}\left(x_{n}\right)$ can be rewritten by substituting

$$
h_{n} \int_{0}^{\infty}\left(x_{n}-u\right) \mathrm{d} F(u)=h_{n}\left(x_{n}-\mu\right) \text { for } n=1, \ldots, N .
$$

This transformation of traditional costs into echelon cost functions proves to be fundamental in decomposing the system. We have (cf. theorem 3.1 in [7]):

Theorem 2.1. Consider a policy which, at the beginning of every period increases the echelon inventory position of echelon $n$ to $y_{n}(n=1,2, \ldots, N)$. Let $D^{(N)}\left(y_{1}, y_{2}, \ldots, y_{n}\right)$ be the associated average costs (which is defined only on $\left\{\left(y_{1} y_{2}, \ldots, y_{n}\right) \mid\left(y_{1} \leqslant y_{2} \leqslant \ldots \leqslant y_{N}\right\}\right)$. We have

$$
D^{(N)}\left(y_{1}, y_{2}, \ldots, y_{N}\right)=C_{1}\left(y_{1}\right)+\ldots+C_{N}\left(y_{1}, \ldots, y_{N}\right)
$$

where 


$$
\begin{aligned}
& C_{1}\left(y_{1}\right)=\int_{0}^{\infty} L_{1}\left(y_{1}-u\right) \mathrm{d} F_{h}(u) \\
& C_{n}\left(y_{1}, \ldots y_{n}\right)= \int_{0}^{\infty} L_{n}\left(y_{n}-u\right) \mathrm{d} F_{l n}(u) \\
&+\int_{y_{n}-y_{n-1}}^{\infty}\left[C_{n-1}\left(y_{1}, \ldots, y_{n-2}, y_{n}-u\right)-C_{n-1}\left(y_{1}, \ldots, y_{n-2}, y_{n-1}\right)\right] \mathrm{d} F_{l_{n}}(u) \text { for } n=2, \ldots, N .
\end{aligned}
$$

Note that $C_{1}\left(y_{1}\right)$ represents the expected costs $l_{1}+1$ periods ahead as a function of the order-up-to level $y_{1}$, i.e.

$C_{1}\left(y_{1}\right)=h_{1}\left(y_{1}-\left(l_{1}+1\right) \mu\right)+\left(p+\sum_{n=1}^{N}\left(h_{n}\right)\right) \int_{y_{1}}^{\infty}\left(u-y_{1}\right) \mathrm{d} F_{1+1}(u)$

Similarly, the first term of $C_{n}\left(y_{n}\right)$ can be rewritten as

$\int_{0}^{\infty} L_{n}\left(y_{n}-u\right) \mathrm{d} F_{l_{n}}(u)=h_{n}\left(y_{n}-\left(l_{n}+1\right) \mu\right)$ for $n=2, \ldots, N$.

The second term in the definition of $C_{n}$ is in fact the extra penalty incurred by installation $n$ due to its unability to completely satisfy the demand of installation $n-1$. The restriction to the area $\left\{\left(y_{1}, y_{2}, \ldots, y_{n}\right) \mid y_{1} \leqslant y_{2} \leqslant \ldots \leqslant y_{N}\right\}$ reflects the fact that the echelon inventory position of installation $n$ can never be smaller than she echelon inventory position of installation $n-1$. Now define for convenience $D^{(n)}\left(y_{1}, \ldots, y_{n}\right)=C_{1}\left(y_{1}\right)+\ldots+C_{n}\left(y_{1}, \ldots, y_{n}\right) \quad n=1,2, \ldots, N$.

Then the following procedure yields the global minimum of $D^{(N)}\left(y_{1}, \ldots, y_{N}\right)$ in the area $\left\{\left(y_{1}, y_{2}, \ldots, y_{n}\right) \mid y_{1} \leqslant y_{2} \leqslant \ldots \leqslant y_{N}\right\}$.

Step 1. Initialization $n:=1$. Minimize $D^{(1)}\left(y_{1}\right)$. Let $S_{1}$ denote the value that minimizes $D^{(1)}\left(y_{1}\right)$.

Step 2. $n:=n+1$. If $n>N$ stop.

Let $\left(S_{1, \ldots,} S_{n-1}\right)$ minimize $D^{(n-1)}\left(y_{1}, \ldots, y_{n-1}\right)$. Minimize next $D^{(n)}\left(S_{1}, \ldots, S_{n-1}, y_{n}\right)$ and let $S_{n}$ denote the corresponding minimizing value. If $S_{n} \geqslant S_{n-1}$ then repeat step 2.

Step 3. Let $k$ be the smallest index such that $S_{k}>S_{n}$. Set $S_{m}:=S_{n}$ for $m=k, \ldots, n$. Goto step 2 .

Hence, the global minimum of $D^{(N)}\left(y_{1}, \ldots, y_{N}\right)$ can be found by subsequently minimizing a number of one-dimensional functions. The proof of this statement is based on the convexity of the functions $D^{(n)}\left(S_{1}, \ldots, S_{n-1}, y_{n}\right)(n=1, \ldots, N)$. Moreover, it can be shown that the average cost optimal policy is contained in the class of policies mentioned in Theorem 2.1., and hence that this average cost optimal policy corresponds with the minimum of $D^{(N)}\left(y_{1}, \ldots, y_{N}\right.$. This is summarized in the following theorem [7]:

Theorem 2.2. The procedure outlined above yields the global minimum of $D^{(N)}\left(y_{1}, \ldots, y_{N}\right)$ in a finite number of steps. The associated policy (which in every period increases the echelon inventory position of echelon $n$ to $S_{n}$ ) is average cost optimal for the infinite horizon problem.

This concludes our review of basic results for serial systems. An immediate extension to assembly systems is briefly discussed in section 6 (see also (71). Furthen uncre, we note that similar results can be established for distribution systems (under a so-called balance assumption which states that, if a 
central depot is unable to satisfy all local warehouse demand, it is still possible to supply these warehouses such that they face equal stockout probabilities, see also [4]). An analysis along the same lines as given above can be found in [7]. Since the average cost functions arising in these systems are considerably more complex they will be treated separately in a forthcoming paper.

Both for serial and assembly systems, the above reviewed decomposition results leave us with the problem to minimize one-dimensional convex functions. Unfortunately, these functions are only recursively defined and involve incomplete convolutions of distribution functions (as will be explained in detail in the forthcoming sections). Therefore, the minimization of these functions is far from trivial. The rest of this paper is devoted to the development of (fast) procedures for solving these minimization problems. Combined with the above outlined decompositions, these procedures enable us to determine optimal policies in fairly complex networks in just a few seconds on a microcomputer.

\section{Approximations of distributions and incomplete convolutions}

This section is devoted to a discussion of the use of mixtures of Erlang distributions to represent the decrease in echelon stock for several echelons, over periods of different length. The choice of mixtures of Erlang distributions is based on several arguments:

- The Normal distribution, often chosen in the literature, does not suit the situations where the coefficient of variation of demand is larger than 0.5 .

- The class of mixture of Erlang distributions is closed under a large number of operations (e.g. convolutions). In particular, the incomplete couvolution of two mixtures of Erlang distributions with the same scale parameter, $\lambda$ say, is again a mixture of Erlang distributions with the same parameter $\lambda$. Incomplete convolutions of mixtures of Erlang distributions can therefore be determined exactly, at least in principle. These incomplete convolutions appear to play a fundamental role in the analysis of multi-echelon systems and will be discussed extensively in this and the forthcoming sections.

- The class of mixtures of Erlang distributions is dense in the set of all possible distributions. In fact this property even holds for the class of mixtures of Erlang distributions with the same scale parameter (see e.g. Schassberger [12]). Therefore, these mixtures can be used effectively to approximately any arbitrary distribution function.

The second argument gives us the opportunity to test the approximations developed in this section azainst an exact procedure. In particular, the cost functions $D^{(N)}\left(y_{1}, y_{2}, \ldots, y_{N}\right)$ can be written in terms of incomplete convolutions of mixtures of Erlang distributions with the same scale parameter and therefore allow for an exact evaluation (cf. Section 4). Unfortunately, this exact evaluation may result in tedious computation schemes; therefore quick approximations are developed in this paper. However, the accuracy of these approximations, reported in Section 5, has been tested against the exact computation schemes, made possible by exploiting the second property above. The need of this property in particularly also rules out the possible use of the Norrnal or the Gamma distribution (often encountered in the literature.

In between, we note the similarity with the analysis by Graves [13] of a distribution system with discrete demands in the METRIC framework (in which he uses a Negative Binomial distribution, i.e. the discrete equivalent of a single Erlang distribution). See also Svoronos and Zipkin [14] for a related model.

Let us now discuss two moment approximations of distribution functions on the nonnegative real axis.Hence, let $x$ be a random variable with distribution function $F(x)$, where we assume $F(x)=0$ for $x \leqslant 0$. Let the first two moments of $x$ be given by $\mu_{1, x}$ and $\mu_{2, x}$, respectively. Define the coefficient of variation $c_{\mathrm{r}}$ by

$c_{x}^{2}=\left\{\mu_{2, r}-\mu_{1, .}^{2}\right\} / \mu_{1, x}^{2}$.

If $c_{*}^{2} \leqslant 1$ then we approximate the distribution function of $x$ by a mixture of an Erlang- $(k-1)$ and an Erlang- $k$ distribution (which is unimodal), which has probability density 
$f(x)=p \lambda^{h-1} \frac{x^{h-2}}{(k-2) !} \mathrm{e}^{-\lambda x}+(1-p) \lambda^{k} \frac{x^{h-1}}{(k-1) !} \mathrm{e}^{-\lambda x}$

with $k$ chosen such that $1 / k \leqslant c^{2} \leqslant 1 /(k-1)$, and $p$ and $\lambda$ defined by

$p=\left[k c_{r}^{2}-\left\{k\left(1+c_{1}^{2}\right)-k^{2} c_{1}^{2}\right\}^{1 / 2}\right] /\left(1+c_{r}^{2}\right)$,

$\lambda=(k-p) / \mu_{1, r}$.

If $c_{r}^{2}>1$ then we approximate the distribution function of $x$ with a hyperexponential distribution with the following density function:

$f(x)=p \lambda_{1} \mathrm{e}^{-\lambda_{1} \alpha}+(1-p) \lambda_{2} \mathrm{e}^{-\lambda_{2} x}$

with $\lambda_{1}, \lambda_{1}$ and $p$ defined by

$$
\begin{aligned}
\lambda_{1} & =\left\{2 / \mu_{1,1}\right\}\left\{1+\left[\left(c_{1}^{2}-0.5\right) /\left(c_{r}^{2}+1\right)\right]^{1 / 2}\right\}, \\
\lambda_{2} & =\left(4 / \mu_{1,1}\right)-\lambda_{1}, \\
p & =\lambda_{1}\left(\lambda_{2} \mu_{1,1}-1\right) /\left(\lambda_{2}-\lambda_{1}\right) .
\end{aligned}
$$

If the original distribution is a Gamma distribution then the hyperexponential fit has even the same first three moments as this original distribution. The above results are easily verified (cf. [9, pp. 397-4001).

Now define for $a \geqslant 0$ the distribution $F^{a}(y)$ by

$$
\begin{aligned}
\lambda_{1} & =\left\{2 / \mu_{1,1}\right\}\left\{1+\left[\left(c_{1}^{2}-0.5\right) /\left(c_{r}^{2}+1\right)\right]^{1 / 2}\right\}, \\
\lambda_{2} & =\left(4 / \mu_{1,1}\right)-\lambda_{1}, \\
p & =\lambda_{1}\left(\lambda_{2} \mu_{1, r}-1\right) /\left(\lambda_{2}-\lambda_{1}\right) .
\end{aligned}
$$

If the original distribution is a Gamma distribution then the hyperexponential fit has even the same first three moments as this original distribution. The above results are easily verified icf. [9, pp. 3974001).

Now define for $a \geqslant 0$ the distribution $F^{a}(y)$ by

$$
\begin{array}{ll}
F^{a}(x)=F(x+a) & \text { if } x \geqslant 0, \\
F^{a}(x)=0 & \text { if } x<0 .
\end{array}
$$

In the next section we will have to evaluate distributions of the form $F^{a} * G$, where $G$ is a continuous distribution function on $[0, \infty)$ with $G(0)=0$, and * denotes the usual convolution operator of the distribution functions of two independent random variables. The function $\left(F^{a} * G\right)(x)$ is also called an incomplete convolution because of the alternative representation.

$$
\left(F^{a} * G\right)(x)=\int_{0}^{x} F(x+a-u) \mathrm{d} G(u),
$$

which is easily shown by partial integration and by exploiting the definition of $F^{a}(x)$. In order to evaluate these incomplete convolutions we now proceed as follows.

Consider first the case in which $c_{r}^{2} \leqslant 1$ and let $F(x)$ be approximated by an appropriately chosen mixture of an Erlang- $(k-1)$ and an Erlang- $k$ distribution. Then it is not hard to verify that the first two moments of the stochastic variable with distribution function $F^{a}, x^{a}$ say, can be approximated by 
$\mu_{1, r^{u}}=p A(k-1, \lambda, a)+(1-p) A(k, \lambda, a)$,

$\mu_{2,1 "}=p B(k-1, \lambda, a)+(1-p) B(k, \lambda, a)$,

where $A(k, \lambda, a)$ and $B(k, \lambda, a)$ are given by

$A(k, \lambda, a)=-a\left(1-G_{h-1}(a)\right)+(k / \lambda)\left(1-G_{h}(a)\right)$,

$B(k, \lambda, a)=a^{2}\left(1-G_{k-2}(a)\right)-(2 a k / \lambda)\left(1-G_{k-1}(a)\right)+\left(k(k+1) / \lambda^{2}\right)\left(1-G_{k}(a)\right)$

and $G_{h}(a)$ the Erlang- $k$ distribution function, i.e.

$G_{h}(a)=1-\sum_{j=0}^{h-1} \mathrm{e}^{-\lambda a} \frac{(\lambda a)^{\jmath}}{j !}$ for $a \geqslant 0$.

If on the other hand $c_{?}^{2}>1$ and $F(x)$ is approximated by a hyperexponential distribution, the first two moments of $\mathrm{x}^{u}$ are approximated by

$\mu_{1, u^{a}}=\left(p / \lambda_{1}\right) e^{-\lambda_{1 a}}+\left((1-p) / \lambda_{2}\right) \mathrm{e}^{-\lambda_{2} a}$,

$\mu_{2,3 a}=\left(2 p / \lambda_{1}^{2}\right) \mathrm{e}^{-\lambda_{1} a}+\left(2(1-p) / \lambda_{2}^{2}\right) \mathrm{e}^{-\lambda_{2} a}$.

From these two moments and the first two moments of the random variable with distribution function $G, y$ say, the first two moments of the convolution of $x^{a}$ and $y$ is derived immediately. Note that this latter random variable is again continuous, with distribution function $F^{a} * G$ while $\left(F^{a} * G\right)(0)=0$. We now again fit a mixture of two Erlang distributions with the same scale parameter or a hyperexponential distribution to the first two moments of $F^{a} * G$ (which have just been calculated) and use this approximation instead of the true distribution $F^{a} * G$.

The procedure just described was developed initially by De Kok and Seidel [10], to study two echelon distribution systems under a service constraint. Four our purposes, i.e. the use in longer serial and assembly systems, the procedure has been used to define an approximation scheme for distribution functions of the type

$\left.\left(F_{l n}^{a_{n}} * F_{l n-1}\right)^{a_{n-1}} * F_{l n-2}\right)^{\left.a_{n-2} * \ldots * F_{l 2}\right)^{a_{2}} * F_{11+1}(x)}$

which appear to arise naturally in multi-echelon serial systems (where the $a_{i}$ 's are positive real numbers and the $l_{1}$ 's are leadtimes again). The approximation scheme works as follows.

From the first two moments of $F_{l n}$ and the approximation by means of either a mixture of Erlang distributions with the same scale parameter or a hyperexponential distribution we may calculate the first two moments of $F_{l n}^{a_{n}}$ and hence of $F_{l_{n}}^{a_{n}} * F_{l_{n-1}}$. After approximating this latter distribution function again we may calculate the first two moments of $\left(F_{t_{n}}^{a_{n}} * F_{l_{n-1}}\right)^{a_{n-1}}$, etc. This approximation scheme has been tested extensively, with very satisfactory results ( $\mathrm{cf}$. Section 5 ). The above procedure will be used extensively in the next two sections, in which we return to multi-echelon production systems.

\section{The two-echelon serial system}

In order to demonstrate the calculation of the optimal control strategies in a multi-echelon system we first analyze the serial system consisting of two iistallations. Recall that the optimal order-up-to levels $S_{1} \ldots S_{2}$ have to be found by minimizing

$D\left(y_{1}, y_{2}\right)=C_{1}\left(y_{1}\right)+C_{2}\left(y_{1}, y_{2}\right)$.

and that these values $S_{1}$ and $S_{2}$ are also the minimizing values of $C_{1}\left(y_{1}\right)$ and $C_{2}\left(S_{1}, y_{2}\right)$, respectively. It is not hard to verify ([7]) that (note that $F_{/ 1+1}(\mathrm{y})=0$ and $F_{/ 2}(y)=0$ for $y<0$ ): 
$C_{1}^{\prime}\left(y_{1}\right)=\frac{\partial}{\partial y_{1}} C_{1}\left(y_{1}\right)=h_{1}-\left(h_{1}+h_{2}+p\right)\left(1-F_{1+1}\left(y_{1}\right)\right)$,

$\frac{\partial}{\partial y_{2}} C_{2}\left(S_{1}, y_{2}\right)=h_{2}+\int_{v_{2}}^{\infty} C_{s_{1}}^{\prime}\left(y_{2}-u_{l_{2}}\right) \mathrm{d} F_{l_{2}}\left(u_{l_{2}}\right)$.

A further investigation of these expressions leads to

Lemma 4.1. Let $S_{1}$ be the minimizing value of $C_{1}\left(y_{1}\right)$. Then

$\frac{\partial}{\partial y_{2}} C_{2}\left(S_{1}, y_{2}\right)=\left(h_{1}+h_{2}\right)-\left(h_{1}+h_{2}+p\right)\left(1-\left(F_{2}^{z_{2}-s_{1}} * F_{1+1}\right)\left(S_{1}\right)\right)$ for $y_{2} \geqslant S_{1}$,

$\frac{\partial}{\partial y_{2}} C_{2}\left(S_{1}, y_{2}\right)=\left(h_{1}+h_{2}\right)-\left(h_{1}+h_{2}+p\right)\left(1-\left(F_{l_{2}} * F_{h_{1}+1}\right)\left(y_{2}\right)\right)$ for $y_{2}<S_{1}$.

Proof. For $y_{2}<S_{1}$ we have

$$
\begin{aligned}
\frac{\partial}{\partial y_{2}} C_{2}\left(S_{1}, y_{2}\right) & =h_{2}+\int_{0}^{\infty} C_{1}^{\prime}\left(y_{2}-u_{l_{2}}\right) \mathrm{d} F_{l_{2}}\left(u_{l_{2}}\right) \\
& =\left(h_{2}+h_{1}\right)-\left(h_{1}+h_{2}+p\right)+\left(h_{1}+h_{2}+p\right) \int_{0}^{y_{2}} F_{l_{1}+1}\left(y_{2} \cdots u_{l_{2}}\right) \mathrm{d} F_{l_{2}}\left(u_{l_{2}}\right) \\
& =\left(h_{1}+h_{2}\right)-\left(h_{1}+h_{2}+p\right)\left[1-\left(F_{l_{2}} * F_{l_{1}+1}\right)\left(y_{2}\right)\right] .
\end{aligned}
$$

For $y_{2} \geqslant S_{1}$ we may write

$$
\begin{aligned}
\frac{\partial}{\partial y_{2}} C_{2}\left(S_{1}, y_{2}\right) & \left.=h_{2}+\int_{v_{2}-s_{1}}^{\infty} C_{1}^{\prime}\left(y_{2}-u_{l_{2}}\right) \mathrm{d} F_{l_{2}}\left(u_{l_{2}}\right) \quad \text { (use } \hat{u}_{l_{2}}=u_{l_{2}}-\left(y_{2}-S_{1}\right)\right) \\
& =h_{2}+\int_{0}^{\infty} C_{1}^{\prime}\left(S_{1}-\hat{u}_{l_{2}}\right) \mathrm{d} F_{l_{2}}\left(\hat{u}_{l_{2}}+\left(y_{2}-S_{1}\right)\right) \\
& \left.=h_{2}+\int_{0^{+}}^{\infty} C_{1}^{\prime}\left(S_{1}-\hat{u}_{l_{2}}\right) \mathrm{d} F_{l_{2}}^{y_{2}-s_{1}}\left(\hat{u}_{l_{2}}\right) \quad \text { (use } C_{1}^{\prime}\left(S_{1}\right)=0\right) \\
& =h_{2}+\int_{0^{-}}^{\infty} C_{1}^{\prime}\left(S_{1}-\hat{u}_{l_{2}}\right) \mathrm{d} F_{l_{2}}^{y_{2}-S_{1}}\left(\hat{u}_{l_{2}}\right) \\
& =\left(h_{2}+h_{1}\right)-\left(h_{1}+h_{2}+p\right)+\left(h_{1}+h_{2}+p\right) \int_{0}^{s_{1}} F_{l_{1}+1}\left(S_{1}-\hat{u}_{l_{2}}\right) \mathrm{d} F_{l_{2}}^{y_{2}-s_{1}}\left(\hat{u}_{l_{2}}\right) \\
& =\left(h_{1}+h_{2}\right)-\left(h_{1}+h_{2}+p\right)\left[1-\left(F_{2}^{y_{2}-s_{1}} * F_{l_{1}+1}\right)\left(S_{1}\right)\right] .
\end{aligned}
$$

It is easily seen from lemma 4.1 . that $\partial C_{2}\left(S_{1}, y_{2}\right) / \partial y_{2}$ is indeed a continuous, monotone non-decreas- 
ing function of $y_{2}$. Next we show how to calculate the order-up-to levels $S_{1}$ and $S_{2}$ from (4.1) and (4.2). We use the procedure given in Section 2, where $\partial D^{(1)}\left(y_{1}\right) / \partial y_{1}=C_{1}\left(y_{1}\right)$ and $\partial D^{(2)}\left(S_{1}, y_{2}\right) / \partial y_{2}$ $=\partial C_{2}\left(S_{1}, y_{2}\right) / \partial y_{2}$. If $F_{1+1}\left(y_{1}\right)$ is given we determine $S_{1}$ directly from $C_{1}^{\prime}\left(y_{1}\right)=0$ (using (4.1)), otherwise we match either a mixture of Erlang distributions with the same scale parameter or a hyperexponential distribution to the first two moments of the $\left(l_{1}+1\right)$-period demand (compare Section 3 ). Call this approximative distribution $G_{11+1}\left(y_{1}\right)$ and determined next $S_{1}$ from $C_{1}^{\prime}\left(y_{1}\right)=0$ with $F_{t_{1}+1}\left(y_{1}\right)$ replaced by $G_{l+1}\left(y_{1}\right)$.

To calculate $S_{2}$ we first determine the sign of $\partial C_{2}\left(S_{1}, S_{1}\right) / \partial y_{2}$ (use (4.2.b)). If $\partial C_{2}\left(S_{1}, S_{1}\right) / \partial y_{2} \geqslant 0$, we have $y_{2} \leqslant S_{1}$ and we may solve the equation

$\left(h_{1}+h_{2}\right)-\left(h_{1}+h_{2}+p\right)\left[1-\left(F_{/ 2} * F_{/ 1+1}\right)\left(y_{2}\right)\right]=0$

to obtain $S_{2}$ (where we again may use an approximative distribution). If however $\partial C_{2}\left(S_{1}, S_{1}\right) / \partial y_{2}<0$ then we have to determine $S_{2}$ from

$\left(h_{1}+h_{2}\right)-\left(h_{1}+h_{2}+p\right)\left[1-\left(F_{22}^{z_{2}-S_{1}} * F_{11+1}\right)\left(S_{1}\right)\right]=0$

(with $y_{2}$ the unknown parameter) by using a bisection method (note that $\left(F_{12}^{v_{2}-S_{1}} * F_{1+1}\right)\left(S_{1}\right)$ is monotone in $y_{2}$ ). In each bisection step (i.e. for every choice of $y_{2}$ ), we fit a distribution to the first two moments of $F_{2}^{\gamma_{2}-s_{1}} * F_{1+1}$ by usilig the approximation described in Section 3. This bisection method finally yields the solution $y_{2}=S_{2}$. The (approximately) optimal policy is now the policy characterized by the parameters $\left(S_{1}, S_{2}\right)$.

For completeness, we also express $D^{(2)}\left(S_{1}, S_{2}\right)$ in terms of $F_{l_{2}}^{S_{2}-S_{1}}$ and $F_{l_{1}+1}$. Define $\hat{B}_{2}(x):=F_{l_{2}+1}(x)$ and $\hat{B}_{1}(x):=\left(F_{l_{2}}^{S_{2}-S_{1}} * F_{l_{1}+1}\right)(x)$. Then we have

Lemma 4.2. The average cost function $D^{(2)}\left(S_{1}, S_{2}\right)$ satisfies

$D^{(2)}\left(S_{1}, S_{2}\right)=\sum_{n=1}^{2}\left(h_{n}\right) \int_{0}^{\infty}\left(S_{n}-u\right) \mathrm{d} \hat{B}_{n}(u)+\left(p+h_{1}+h_{2}\right) \int_{S_{1}}^{\infty}\left(u-S_{1}\right) \mathrm{d} \hat{B}_{1}(u)$

Proof. By applying the same transformation as in the last part of the proof of lemma 4.1 it is easy to show that

$D^{(2)}\left(S_{1}, S_{2}\right)=h_{2}\left(\int_{0}^{\infty}\left(S_{2}-u_{t+1}\right) \mathrm{d} F_{l^{2}+1}\left(u_{l_{2}+1}\right)\right)+\int_{0^{-}}^{\infty} C_{1}\left(S_{1}-\hat{u}_{l_{2}}\right) \mathrm{d} F_{l_{2}}^{S_{2}-S_{1}}\left(\hat{u}_{l_{2}}\right)$.

For the second expression at the right hand side of this equation we may write

$$
\begin{aligned}
& \int_{0-}^{\infty} C_{1}\left(S_{1}-\hat{u}_{l_{2}}\right) \mathrm{d} F_{l_{2}}^{S_{2}-S_{1}}\left(\dot{u}_{l_{2}}\right) \\
& \quad=h_{1} \int_{0^{-}}^{\infty}\left(S_{1}-\hat{u}\right) \mathrm{d}\left(F_{l_{2}}^{S_{2}-S_{1}} * F_{l^{+}+1}\right)(u)+\left(p+h_{1}+h_{2}\right) \int_{S_{1}}^{\infty}\left(u-S_{1}\right) \mathrm{d}\left(F_{l_{2}}^{S_{2}-S_{1}} * F_{l_{1}+1}\right)(u)
\end{aligned}
$$

From the definitions of $\hat{B}_{2}(x)$ and $\hat{B}_{1}(x)$ the result now folluws immediately.

In all cases tested the resulting values of $S_{2}$ deviated less than $1 \%$ from the value found by an exact method (briefly discussed in Section 5). Hence, the approximation procedure described in Section 3 yields results accurate enough for our purposes. However, the results can even be improved by applying three moment fits. Details are left to the reater. More extensive numerical results will be reported at the end of the next section. 


\section{Analysis of general serial produetion systems}

In this section we show how to calculate optimal order-up-to levels in general serial systems. Consider a N-echelon serial system as described in Section 2 , with leadtimes $I_{N} I_{N-1}, \ldots, I_{1}$. Define

$I I(y)=F_{h+1}(y)$,

$H_{i}^{\prime}(y)=\left(F_{l_{n}} * \ldots * F_{h_{2}} * F_{h_{1}+1}\right)(y)$ for $n \geqslant 2$

and for $2 \leqslant k \leqslant n$ (with again $n \geqslant 2$ )

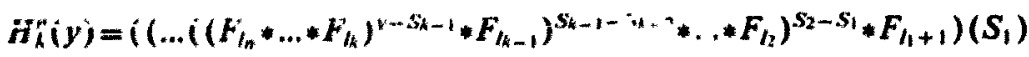

where * denoirs the convolution operator again. Finally, let

$J_{h}^{n}(y)=\sum_{j=1}^{n} h_{i}-\left(p+\sum_{j=1}^{N} h_{i}\right)\left(1-H_{k}^{n}(y)\right)$

The following generalization of lemma 4.1 is stated without proof:

Theorem 5.1. Let, for each $n$ with $1 \leqslant n \leqslant N, D^{(n-1)}\left(y_{1}, \ldots, y_{n-1}\right)$ take its absolute minimum value in $\left(S_{1}, S_{2}, \ldots, S_{n-1}\right)$. Then the partial derivatives of $D^{(n)}\left(S_{1}, \ldots, S_{n-1}, y_{n}\right)$ to $y_{n}$ satisfy

$\frac{\partial}{\partial y_{n}} D^{(n)}\left(S_{1}, \ldots, S_{n-1}, y_{n}\right)=J_{n}^{n}\left(y_{n}\right) \quad$ for $y_{n}>S_{n-1}$,

$\frac{\partial}{\partial y_{n}} D^{(n)}\left(S_{1} \ldots ., S_{n+1}, y_{n}\right)=J_{k}^{n}\left(y_{n}\right) \quad$ for $S_{k-1}<y_{n} \leqslant S_{k}, k=2, \ldots, n-1$,

$\frac{\partial}{\partial y_{n}} D^{(n)}\left(S_{1}, \ldots, S_{n-1}, y_{n}\right)=J_{1}^{n}\left(y_{n}\right) \quad$ for $y_{n} \leqslant S_{1}$.

Theorem 5.1 is easily proved by induction. Hence, the procedure described in Section 2 can be applied to determine the values $S_{1}, S_{2}, \ldots, S_{N}$ if we are able to evaluate the functions $J_{k}^{n}\left(y_{n}\right)$ or $H_{k}^{n}\left(y_{n}\right)$. However, for each value of $y_{n}$ we may approximate $H_{k}^{n}\left(y_{n}\right)$ by successive fits of mixtures of Erlang distributions with the same scale parameter (or hyperexponential distributions) as described at the end of Section 3. Below, we describe how to select successive trial values of $y_{n}$.

Suppose we wish to det?:mine $y_{n}=S_{n}$ as the solution of

$\frac{\partial}{\partial y_{n}} D^{(n)}\left(S_{1}, \ldots, S_{n}, \quad, y_{t}\right)=0$,

where we now suppose $n \geqslant 2$ (the case $n=1$ is easy, we simply have to solve $J \mid(y)=0$ ). The following procedure yields $S_{n}$.

Step 1. Set $k=n$.

Step 2. Determine $H_{k}^{n}\left(S_{k-1}\right)$ and subsequently $J_{k}^{n}\left(S_{k-1}\right)$. If $J_{k}^{n}\left(S_{k-1}\right)<0$ then go to Step 3, otherwise set $k:=k-1$, If $k=1$ then goto Step 3, otherwise repeat Step 2 .

Step 3. Apply a bisection method to solve $J_{h}^{n}\left(y_{n}\right)=0$, yielding $y_{n}=S_{n}$. Stop.

If step 3 is entered with $k=n$ then we know that $S_{n}>S_{n-1}$. If $2 \leqslant k<n$ then $S_{k-1}<S_{n} \leqslant S_{k}$ and if $k=1$ then $S_{n} \leqslant S_{1}$. In all cases the right runction $J_{k}^{n}\left(y_{n}\right)$ is evaluated.

The above procedure should be used to execute the second step in the procedure of section 2, for every $n$. This solves the serial system.

For completeness, we also state the analogon of lemma 4.2. Define recursively

$B_{N}(x):=F_{i N}(x)$, 
$B_{n}(x)=\left(\left(B_{n+1}\right)^{s_{n+1}-S_{n}} * F_{l n}\right)(x)$ for $n=N-1, \ldots, 1$.

Finally, we need distribution functions $B_{n}(x)$, defined by

$B_{n}(x)=\left(B_{n} * F\right)(x)$ for $n=N-1, \ldots, 1$.

Using these definitions, the following result is easily shown by induction.

Theorem 5.2. The average cost function $D^{(N)}\left(S_{1}, S_{2}, \ldots, S_{N}\right)$ satisfies

$D^{(N)}\left(S_{1} S_{2}, \ldots, S_{N}\right)=\sum_{n=1}^{N} h_{n} \int_{0}^{\infty}\left(S_{n}-u\right) \mathrm{d} B_{n}(u)+\left(p+\sum_{n=1}^{N} h_{n}\right) \int_{S=1}^{\infty}\left(u-S_{1}\right) \mathrm{d} B_{1}(u)$

The reader may note some similarity between the functions $\hat{B}_{n}$ and the functions $H_{k}^{n}$ defined previously. Indeed we have for exampl: $\hat{B}_{N}\left(S_{j}\right)=H_{N}^{N}\left(S_{N}\right)$. The functions $H_{k}^{n}$ however serve to determine the order-up-to levels $S_{n}$. The functions $B_{n}$ describe the behavior of the system once these order-up-to levels have been determined.

The next tables show the accuracy of the approximation method by comparing it with an exact procedure. This exact procedure is based on the fact that incomplete convolutions can be determined exactly as long as the underlying distributions are of mixtures of Erlang distributions, all with the same scale parameter, $\lambda$ say. Although the formulas and tre cal'mitatiuns become somewhat tedious, it is therefore possible to evaluate functions of the type

$\left.\left(F_{l_{n}}^{a_{n}} * F_{l_{n-1}}\right)^{a_{n-1}} * F_{l_{n-2}}\right)^{\left.a_{n-2} * \ldots * F_{l_{2}}\right)^{a_{2}} * F_{l_{1}+1}(x)}$

as long as the demand distribution is a mixture of Erlang distributions with the same scale parameter $\lambda$ (the same then holds for all (incomplete) convolutions). Hence, also the functions $D^{(11)}\left(S_{1}, \ldots, S_{n-1}, y_{n}\right)$ and their derivatives can be determined exactly. In a forthcoming paper we will discuss in more detail the calculation scherue of this exact procedure.

Table 1 shows results for the case where $N=3, \mu=100, h_{1}=1, h_{2}=3, h_{3}=6, p=200, l_{1}=1, l_{2}=3, l_{3}=2$.

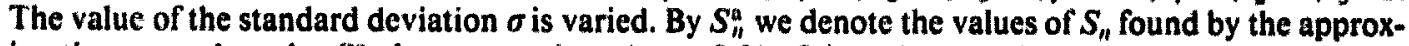
imation procedure, by $S_{n}^{\mathrm{e}}$ the exact values $(n=1,2,3)$. Ctime denotes the computation time of the approximate values on an IBM compatible $\mathrm{PC} / \mathrm{XT}$.

Table 2 is based on the same set of parameters as Table 1, except that now $\sigma=70$ while $p$ has been taken as the varying parameter (changing the ratios between the inventory holding costs and the penalty costs actually means that we are changing a target customer service level, see also Theorem $\mathbf{5 . 3}$ below).

\section{TABLE I}

Approvimate and eyac: *n!ues of $S_{n}(n=1,2,3)$, for varying $\sigma$

\begin{tabular}{|c|c|c|c|c|c|c|c|}
\hline$\sigma$ & $s_{i}^{\prime \prime}$ & $\mathrm{ST}_{\mathrm{i}}$ & $S_{2}$ & $S_{\mathrm{z}}$ & $S_{3}$ & $S_{i}$ & ctime \\
\hline 10 & 2386 & 238.6 & 5463 & 549.1 & 744.2 & 746.6 & $3.2 \mathrm{~s}$ \\
\hline 20 & 280.9 & 280.9 & 595,6 & 600.4 & 790.3 & 794.3 & $0.9 \mathrm{~s}$ \\
\hline 30 & 327,0 & 326.9 & 647.8 & 653.8 & 838.1 & 842.9 & $0.6 \mathrm{~s}$ \\
\hline 40 & 376.5 & 376.2 & 702.3 & 709.1 & 887.5 & 892.3 & $0.4 \mathrm{~s}$ \\
\hline 50 & 4303 & 4303 & 760.6 & 766.9 & 938.1 & 942.8 & $0.3 \mathrm{~s}$ \\
\hline 60 & 485.6 & 485.2 & 820.9 & 825.2 & 989.4 & 993.4 & 0.38 \\
\hline 70 & 546.3 & 546.1 & 881.7 & 886.9 & 1042 & 1045 & $0.3 \mathrm{~s}$ \\
\hline 80 & 608.3 & 602.1 & 947.3 & 945.8 & 1095 & 1096 & $0.2 \mathrm{~s}$ \\
\hline 90 & 670.3 & 666.0 & 1010 & 1009 & 1150 & 1149 & $0.2 \mathrm{~s}$ \\
\hline 100 & 7485 & 748.5 & 1083 & 1081 & 1204 & 1204 & $0.2 \mathrm{~s}$ \\
\hline
\end{tabular}


TABLE 2

Approximule and exacl values of $\$$ (n $1,2,3)$, for varyins $p$

\begin{tabular}{|c|c|c|c|c|c|c|c|}
\hline p & 59 & $\$ 2$ & 5 & 51 & $\$ 1$ & Si & ciuns \\
\hline 40 & 450.7 & 450.6 & 739.7 & 741.7 & 847.4 & 854.2 & $0.3 \mathrm{~s}$ \\
\hline 80 & 490.6 & 490.5 & 801.4 & 804,2 & 935.0 & 938.9 & $0.3 \mathrm{~s}$ \\
\hline 120 & 515.0 & $\$ 14.9$ & 837,3 & 841.0 & 983.7 & 986.9 & $0.3 \mathrm{~s}$ \\
\hline 160 & 532.6 & 532.4 & 862.4 & 866.9 & 1017 & 1020 & $0.3 \mathrm{~s}$ \\
\hline 200 & 546.3 & 546.1 & 881.7 & 886.9 & 1042 & 1045 & $0.3 \mathrm{~s}$ \\
\hline 240 & 557.5 & 5573 & 897.2 & 903.1 & 1062 & 1066 & $0.3 \mathrm{~s}$ \\
\hline 280 & 567.0 & 566.8 & 910.3 & 916.7 & 1078 & 1083 & $0.2 \mathrm{~s}$ \\
\hline 320 & 575.2 & 575.1 & 921.5 & 928.4 & 1092 & 1097 & $0.2 \mathrm{~s}$ \\
\hline 360 & $\$ 82.5$ & 5823 & 931.3 & 938.7 & 1105 & 1110 & $0.2 \mathrm{~s}$ \\
\hline 400 & 589,0 & 588.8 & 940.0 & 947.8 & 1115 & $|12|$ & 0.28 \\
\hline
\end{tabular}

TABLE 3

Parameter settings of examples. investigated in Table 4

\begin{tabular}{|c|c|c|c|c|c|c|c|c|c|c|}
\hline$N$ & $k_{1}$ & $h_{2}$ & $b_{1}$ & $t_{4}$ & $h_{s}$ & $I_{1}$ & $l_{2}$ & $I_{3}$ & $l_{4}$ & ls \\
\hline 1 & 10 & & & & & 5 & & & & \\
\hline 2 & 2 & 8 & & & & $i$ & 4 & & & \\
\hline 3 & 2 & 2 & 6 & & & $i$ & $i$ & 3 & & \\
\hline 4 & 2 & 2 & 2 & 4 & & $i$ & $i$ & $i$ & 2 & \\
\hline 5 & 2 & 2 & 2 & 2 & 2 & 1 & $i$ & $i$ & 1 & 1 \\
\hline
\end{tabular}

TABLE 4

Approximate and exact values of $S_{n *}$ for different chain lengths

\begin{tabular}{|c|c|c|c|c|c|c|c|c|c|c|}
\hline$N$ & $s i q_{i}$ & $S \%$ & $\$ 3$ & 54 & $S_{3}$ & $S q$ & S) & $\mathbf{S Y}$ & $S_{\$}^{*}$ & $S \mathbf{S}$ \\
\hline 1 & 9121 & & & & & 912.1 & & & & \\
\hline 2 & 500.9 & 917.4 & & & & $\$ 00.8$ & 921.7 & & & \\
\hline 3 & $\$ 00.9$ & 6164 & 929.1 & & & 500.8 & 615.8 & 932.8 & & \\
\hline 4 & $\$ 00,9$ & 616.4 & 734.0 & 942.5 & & 500.8 & 615.8 & 733.4 & 945.2 & \\
\hline$\$$ & 500.9 & 616.4 & 734.0 & 846.8 & 957.9 & 500.8 & 615.8 & 733.4 & 848.2 & 960.2 \\
\hline
\end{tabular}

Finally, we investigate the influence of the number of stocks points on the quality of the approximauon. As before, we have $\mu=100, \sigma=80$ and $p=200$. The other parameters are chosen according to Table 3 (where we kept the sum of the inventory holding cost parameters and the leadtimes equal to 10 and 5 , resp.). Results are presented in Table 4. The largest computation time (for $N=5$ ) was equal to 0.6 sec. In sum, we may conclude that the approximation method performs extremely well. Deviations between approximate and exact values of $D^{i N}\left(S_{1}, \ldots, S_{N-1}, S_{N}\right)$ appear to be slightly larger but are never larger than 2\%. If we combine an exact calculation of the latter function with the approximated values $S_{n}^{a}$ the deviation becomes almost negligable (less than $0.1 \%$ ). More extensive test results are available from the author; on request.

\section{Assembly systems and service degree considerations}

Next, we cousider a production system in which $N$ components are assembled into a single end item. Without loss of generality we may assume that only one componint of each type is needed to assemble 
one product. Components are delivered by (infinite capacity) outside suppliers where a supply leadtime $l$, is needed for a component of type $i(l=l, \ldots ., N)$. Let $l_{0}$ denota the assembly leadtime. The fact that final products can be assembled only if components of all types ar $e$ available in sumcient quantities clearly demands for some coordinalion in ordering the different comjionent types.

As before, only final products are subject to outside demand. Compronents in the system (in stock at the component store or as part of work-in-process in the assembly phase) are subject to a holding cost $h_{i}$ (for component type $i$ ), final products are stored at a holding cost $h_{0}+\sum_{n=1}^{N} h_{n}$, while a penalty $p$ is incurred if demand cannot be met immedintely and has to be backlogged. All costs are calculated at the end of a period.

Recall that the echelon stock of a component includes components already assembled in end items stored in the final product warehouse. As in the case of the serial system, there is also for assembly systems a class of policies which deserves special attention. The structure of these policles however is less obvious than in the serial calle and therefore discussed in some detail.

Without loss of generality we may assume $l_{1}<l_{2}<\ldots<l_{N}$ (since all leadtimes are deterministic we may treat components with equal order leadtime as one "aggregate" component). Wo will consider policies that are characterized by so-called potential order-up-to levels $y_{n}$ for cach componsant type $n$ $(n=1, \ldots, N)$. Now suppose that, at the beginning of period $l$, the inventory position of components of type $N$ is increased to $y_{N}$. Let $u_{N-I_{N=1}}$ denote the outside demand (translated in terms of components of type $N$ ) between $t$ and $t+l_{N}-l_{N-1}$. Then it does not make sense to increase the inventory position of components of type $N-1$ to a level higher than $y_{N}-u_{I_{N-I_{N-1}}}$, at time $t+I_{N}-l_{i^{\prime}-1}$, since any larger order would result in temporarily useless stock of components of type $N-1$ at time $l+I_{N}$ (note that the echelon stocks of both component types are subject to the same demand pattern between time $t+I_{N}-I_{N-1}$ and time $t+l_{N}$ ). On the other hand, we may consider an independent order-up-to level $y_{N-1}$ (also called a potential order-up-to level! which serves as an upper bound for the inventory position of component type $N-1$. Therefore, we increase the inventory position of component $N-1$ to $\min \left(y_{N-1}\right.$, $y_{N}-u_{N-I_{N-1}}$ ) at time $t+l_{N}-l_{N-1}$. Note that this corresponds with the decision structure in a two stage serial system with leadtimes $l_{N}-l_{N-1}$ and $l_{N-1}$.

More general, it can be shown that an assembly system with leadtimes $l_{0}$ (for the assembly phase) and $l_{n}$ (for supplying component type $n$ ), $n=1, \ldots, N$, can be analyzed as a serial system with leadtimes $l_{N}-l_{N-1}, \ldots, l_{2}-l_{1}, l_{1}$ and $l_{0}$. The average cost function for the assembly system is not completely identical however to the one for the corresponding serial system, due to the fact that components of type $n$ arrive in the system only after $l_{n}$ periods (instead of anter $l_{n}-l_{n-1}$ periods) which influences the holding cost functions. More precise, if the functions $C_{n}\left(y_{0}, y_{1}, \ldots, y_{n}\right)$ are defined for the serial system with leadtimes $l_{N}-l_{N-1}, \ldots l_{2}-l_{1}, l_{1}$ and $l_{0}$ (compare Theorem 2.1 ) then the corresponding functions $C_{n}\left(y_{0}, y_{1}, \ldots, y_{n}\right)$ for the assembly system satisfy

$C_{n}\left(y_{0}, y_{1}, \ldots, y_{n}\right)=C_{n}\left(y_{0}, y_{1}, \ldots, y_{n}\right)-h_{n} \mu\left(l_{n}-l_{n-1}\right)$

The average cost for the complete assembly system is then simply the sum of the functions $C_{n}\left(y_{0}, y_{1} \ldots, y_{n}\right)$ again. Note that (6.1) : $ı$ mediately implies that

$$
\frac{\partial}{\partial y_{n}} C_{n}\left(y_{0}, y_{1}, \ldots, y_{n-1}, y_{n}\right)=\frac{\partial}{\partial y_{n}} C_{n}\left(y_{0}, y_{1}, \ldots, y_{n-1}, y_{n}\right) \quad n=0,1, \ldots, N \text {. }
$$

As a result, the order-up- $p$ levels in an assembly system can bc found by applying Theorem 5.1 and the approximation scheme nutlined ahove to the serial system with leadtimes $l_{n}-l_{n-1}(n=2 \ldots, N), l_{1}$ and $l_{0}$. In sum, assembly s/stems can be analyzed along the same lines as serial systems with leadtimes adapted appropriately.

We end this section with an indication on how to translate the results obtained so far to models in which we seek to minimize average holding costs under the constraint that a target customer service level (CSL) should be met. Consider first a s" "ial system. Form Theorem 5.2 we immediately deduce: 
Theorem 6.1. Let the customer service level $\delta$ be definect as the fraction of demand satiafied direc lly from stock (at installation 1 ) under the average cost optimal policy $\left(S_{1}, S_{\xi+*}, S_{N}\right)$. Then $\delta$ satisfies

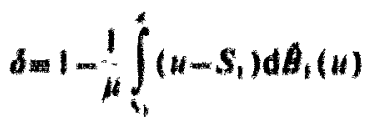

where $\mu$ denoles the expected one-period demand.

It is intuitively clear (and it can be proved rigorously) that $\delta$ is a monotone increasing function of the unit penalty cost $p$, and vice versa. Since we have developed quick minimization procedures for the analysis of serial systems, it is easy to fit a penalty cost p such that a target customer service level $\tau$ is met (for example, by using bisection ). As a result we then have found a policy, satisfying a target CSL, which is average cost optimal with respect to the sum of inventory holding and (associated) penalty costs.

The remaining question now is whether this policy is also optimal with respect to minimizing the average inventory holding cosis solely, within the class of all strategies satisfying a tarect customer service level (CSL). Fortunately, the following (general) result is casily established:

Theerem 6.2. I et $\Gamma$ denote an average cost optumal strategy with respect to the sum of inventory holding and penalty costs, where the unit penalty cost $p$ is chosen such that $\Gamma$ satisfies a target CSL, $\tau$ say. Then $r$ is also optimal in the sense that, within the class of all strategits satisfying the service constraint $\tau$, it is a strategy with minimum average inventory holding costs.

Summarizing, for large multi-echelon serial systems we are abie to determine policies, satisfying a target CSL. such that the the averase inventory holding costs are minimized. Note that exact results can be obtained as long as we assume that the demand distributions are mixtures of Erlang distributions with the same scale parameter. The tesults hold for assembly systems as well. due to the indicated correspondence between serial and assembly systems.

\section{Conclustons and suggentions for future research}

In this paper, we have reviewed our theoretical analysis of multi-echelon production systems, in particular the fundamental decomposition results for serial and assembly systems. These (exact) decompositions leave us with the problem to determine numerically order-up-to levels in complex one-dimensional inventory systems. An approximation scheme has been defined to solve this latter problem. For systems which are not too large ( 4 in 5 echelons), numerical tests have shown a highly accurate performance. In particular, the order-up-to levels never deviate more than $1 \%$ from their exact values, thus justifying application of the approximation.

Our first goal will be to extend the approximation scheme to distribution systems as well. Although these systems satisfy similar decomposition properties as serial and assembly systems (but under an additional so-called balance assumption, see the end of section 2 or Langenhof and Zijm (7]), numerical difficulties arise if the central depot is unable to satisfy all demand from the local warehouses. The allocation function appears as the solution of a parametric nonlinear convex minimization problem and is not easily characterized in general, not even under a balance assumption (except for the normal distribution).

A second extension includes the assumption of finite capacities in some phases, both in distribution systems (in the first production phase) as well as in assembly systems (in the latter assembly phase and in some component manufacturing phases). Here we exploit a result of Federgruen and Zipkin (15]) on the optimality of (S.)-policies for single stage inventory systems without fixed costs but with finite 
capacity (under stationary stochastic demand). However, the calculation of the order-up-to levels in finite capacity systems is far from trivial again. It can be shown that the analysis resembles the optimization of certain parameters in a $D / C / 1$ queve with a cont structure (where $G$ may be a mixture of Erlang distributions with the same scale parameter aeain). This will bo the topic of a forthcoming paper.

Further work will include multi-product environments as well as the discusuion of nonutationary demand environments. In particular, we will focus on the combination of ideas from a more capacity oriented approach such as Hierarchical Production Planning (see c.g [16]) and the (stochastic) material coordination appraoch reviewed here.

\section{Acknowledgement}

The authors wish to express their gratitude to $\mathrm{Dr}$. K, Rosling for muny eonstruetive remarks which have led $t 0$ a major improvement of the original paper. Two anonymous referees are also gratefully acknowledged.

\section{References}

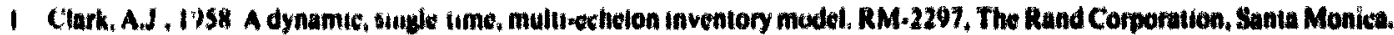

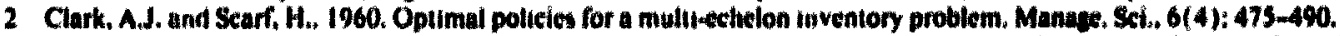

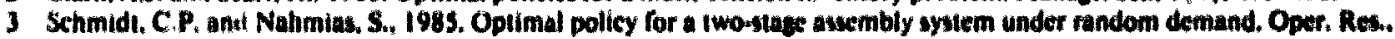
$33(5): 1100-1145$.

4 Eppen. O. and Sehrage, $L, 1981$. Centralized ordering policies in a muliwarehouse system wim lead times and nadom demand. In: Skhwark, L., (Ed.), Multi-ievel Production/Inventory Controi systems Theory and Pruetice (Studies in the Managemeat Seiences, Vol. 16). North-Holland, Amsterdam, pp. 51-67.

5 Federgruen, A. and Zipkin. P. 1984, Approximations of dynamic, multilocition production and inventory problems. Menase. Sct., 30(1):69-84,

6 Federgruen, A. and Zipkin. P.. 1984. Compulational issues in an infinite-horimn. wultiechelon inventory model. Oper, Ret. 32(4): 818-836.

7 Lungenholf, L.J.G. and Zum, W.H.M., 1990. An analytical theory of multecheton production/diunibnition sytems, Statustica Neerlandica, 44 (3): 149-174.

8 Rosling. K. 1989. Optimal inventory polictes for astembly systems under randorn demanda. Oper. Res. 37 (4) 365 - 579.

9 Tums, H.C, 1986. Stochastic Modelling and Andycis: A Computationd Approach Wiley, Now York.

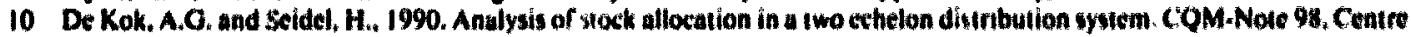
for Quantitative Methods, Philips Electronics Eindlioven.

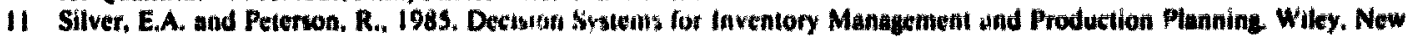
York, 2nd edn.

12 Schaseberger, R.. 1973. Wancsehlanghn. springet, Uetha.

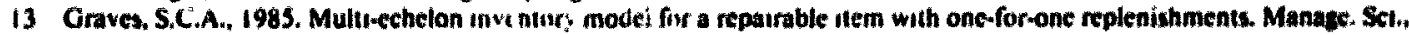
$31(10), 1247-1256$.

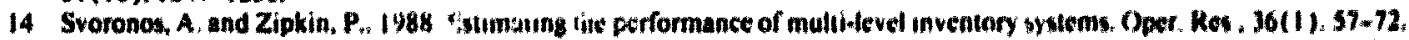

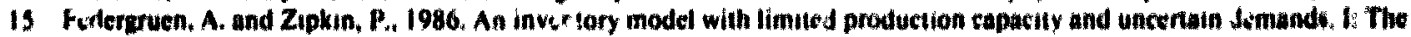
average cost criterion. math. Oper. Res, $11(2): 103-20 \%$.

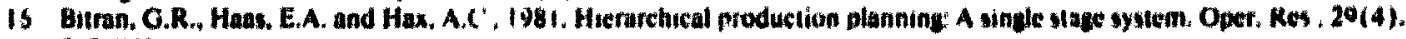
717-743. 